\title{
A New Approach in Defining Harmonic Indices in Power Utility Application
}

\author{
H. Mokhtari and A. Shahab and M. Poshtan \\ ${ }^{1}$ EE Department \\ Sharif University of Technology \\ Tehran, Iran, mokhtari@sharif.edu \\ ${ }^{2}$ EECE Department \\ AMERICAN UNIVERSITY IN DUBAI \\ Dubai, UAE \\ Phone: +97143183415, email: mposhtan@ aud.edu
}

\begin{abstract}
This paper proposes a new method for current harmonic evaluation. Several harmonic indices have been defined for this purpose among which the Total Harmonic Distortion (THD) is the most widely used one. However, all these indices suffer from some deficiencies such as giving the same weight to all harmonic orders, and not giving a clear relation between the harmonics and their effects on system equipment. In this paper, for the first time, an index is defined based on current harmonic effects on transformers and power lines. A new formula is defined in order to determine the share of each harmonic order. The paper also proposes a new harmonic limit for the derived index. The limit is based on the de-rating of a transformer subjected to harmonic environments in order to keep its life equal to that of the same transformer when handles only fundamental current. In order to compare the effectiveness of the new index, several real cases with the same THD level are evaluated using the proposed index.
\end{abstract}

Keywords: - power quality, harmonic index, power system harmonics, and harmonic losses

\section{Introduction}

Increasing growth in the use of non-linear and sensitive equipment has made harmonic studies of great importance. Harmonics have adverse effects on equipment including reduction in efficiency and loss of life. Therefore, it is necessary to evaluate the level of harmonic pollution in real systems. This evaluation must be done based on suitable harmonic indices. The most commonly used index for harmonics is Total Harmonic Distortion or THD, which is defined as:

$$
T H D=\frac{\sqrt{\sum_{h=2}^{h_{\max }} I_{h}^{2}}}{I_{1}}
$$

Where $I_{1}$ and $I_{h}$ are the rms of the fundamental and harmonic component of the current. However, from one hand THD does not differentiate between different harmonics. From the other hand, most of the times, the adverse effect of harmonics on equipment is in relation with the harmonic order. In order to alleviate this problem,
IEEE519 [IEEE, 1992] has set current harmonic limits for individual harmonics as well as for current THD. But there is not much explanations behind IEEE519 limits. Cmessage index tries to differentiate between different harmonics by applying a weighting factor for different harmonics. However, in this index, i.e. C-message, the weighting factor stems from the effect of harmonics on human hearing.

In this paper, a new harmonic index is defined considering the effect of harmonics on system equipment, i.e. transformers and power lines. The proposed index takes into account the share of each harmonic in system losses and consequently loss of life of power system equipment. In doing so, for each harmonic, a new weighting factor is defined. The new factor is proportional to the losses generated due to the harmonic order.

Based on the new harmonic index, proposed here, the limit is based on the reduction in the fundamental component of load current in order not to affect the life of system equipment. In this approach, the life of system transformers and distribution lines is equal to that of the same equipment when they are used in a harmonic-free environment. The structure of this paper is as follows. In section II, a review on different losses in a transformer is given based on IEEE standards C570.110-2008, C57.12.90-2006 [IEEE,1995] and C57.12.912001 [IEEE,1998]. In Section III, a new harmonic index is proposed for current harmonics considering transformer losses based on IEEEC57.110-2008. This section also evaluates the suitability of the new weighting index by applying it to several real measurement cases. Section IV sets the conditions for the new harmonic index when the effects on power lines are taken into consideration.

\section{Transformer Losses Categorized by IEEE Standards}

Transformer losses are divided into the following three categories by IEEE Std. C57.12.90-1993 [IEEE,1995] and IEEE Std. C57.12.91-1995 [IEEE,1998]:

1. No-load losses (excitation losses) (PNL)

2. Load losses (impedance losses) (PLL) 
3. Total loss (the sum of no-load losses and load losses) (Ptot).

Load losses are further divided into ohmic R losses (P) and stray losses. According to [IEEE,1992IEEE,1998], stray losses are due to the electromagnetic flux in different parts of a transformer and can be divided into winding stray losses (PEC) and stray losses in components other than the windings (POSL). Therefore:

$$
P_{L L-R}=P_{R}+P_{E C-R}+P_{O S L-R}
$$

Where PLL-R is the rated value of load losses, PR is the rated value of $\mathrm{R}$ losses, PEC-R is the rated value of winding stray losses, and POSL-R is the rated value of stray losses in components other than windings.

Considering PR losses as the base for power calculations, then in per-unit form:

$$
\begin{aligned}
& P_{L L}(p u)=P(p u)+P_{E C}(p u)+P_{O S L}(p u) \\
& \text { and : } \\
& P_{L L-R}(p u)=1+P_{E C-R}(p u)+P_{O S L-R}(p u)
\end{aligned}
$$

$\mathrm{R}$ losses are proportional to the squares of the rms load current [3]. Therefore:

$$
P(p u)=\sum_{h=1}^{h_{\max }} I_{h}^{2}(p u)
$$

Eddy current loss is proportional to the squares of the rms load current and frequency [IEEE1998]. Therefore:

$$
P_{E C}(p u)=P_{E C-R}(p u) \sum_{h=1}^{h_{\max }} I_{h}^{2}(p u) h^{2}
$$

Where $h_{\max }$ represents the highest harmonic order.

The losses in parts other than windings are proportional to the square of the rms load current and the harmonic order of power 0.8 [IEEE1998], i.e.:

$$
P_{O S L}(p u)=P_{O S L-R}(p u) \sum_{h=1}^{h_{\max }} I_{h}^{2}(p u) h^{0.8}
$$

Harmonic losses cause extra heat in transformers resulting in an increase in the hottest-spot temperature. Therefore, in order to keep the transformer life unchanged, it is necessary to de-rate the transformer when operating in harmonic polluted environment. The maximum current which can be put through a transformer when harmonics are present can be found from [IEEE1998] as:

$$
I_{\max }(p u)=\left(\frac{P_{l l-R}(p u)}{1+F_{H L} \times P_{E C-R}(p u)}\right)^{0.5}
$$

where $F_{H L}$ is the harmonic loss factor for winding eddy currents which is determined by [IEEE1998]:

$$
F_{H L}=\left(\frac{\sum_{h=1}^{h_{\max }}\left[\frac{I_{h}}{I_{1}}\right] h^{2}}{\sum_{h=1}^{h_{\max }}\left[\frac{I_{h}}{I_{1}}\right]}\right)^{0.8}
$$

\section{New Weighting Harmonic Index}

In this section, a new current harmonic index (WTHD) is defined. The new index is a weighting one whose weights are set based on contribution of each harmonic in the transformer losses. The index is first defined for dry-type transformers. It is then checked for liquid-filled transformers.

\section{A. Dry-type Transformers}

According to IEEE Std. C.57.110, the winding hottestspot temperature rise over the ambient temperature is proportional to the load losses to the power of 0.8 .

$$
\theta_{g}=\theta_{g-R}\left(\frac{P_{L L}(p u)}{P_{L L-R}(p u)}\right)^{0.8}
$$

Where $\theta_{g}$ the hottest-spot temperature at working is condition, and $\theta_{g-R}$ is the hottest-spot temperature at rated load.

Since there is no need of considering other stray losses in dry-type transformers, from (3) and (10), one can write:

$$
\theta_{g}=\theta_{g-R}\left(\frac{P(p u)+P_{E C}(p u)}{1+P_{E C-R}(p u)}\right)^{0.8}
$$

Substituting from (5) and (6) into (11) results in:

$$
\theta_{g}=\theta_{g-R}\left(\frac{\sum_{h=1}^{h_{\text {max }}} I_{h}^{2}(p u)+P_{E C-R}(p u) \sum_{h=1}^{h_{\text {max }}} I_{h}^{2}(p u) h^{2}}{1+P_{E C-R}(p u)}\right)^{0.8}
$$

In order to keep a transformer life equal to that of the same transformer operating at rated values, the winding hottest-spot temperature must be held equal to its rated value:

$$
\theta_{g}=\theta_{g-R}
$$

Therefore:

$$
\begin{aligned}
& \left(\frac{P_{L L}(p u)}{P_{L L-R}(p u)}\right)^{0.8}=1 \\
& \left(\frac{\sum_{h=1}^{h_{\max }} I_{h}^{2}(p u)+P_{E C-R}(p u) \sum_{h=1}^{h_{\max }} I_{h}^{2}(p u) h^{2}}{1+P_{E C-R}(p u)}\right)^{0.8}=1
\end{aligned}
$$

Or:

$$
\frac{\sum_{h=1}^{h_{\max }} I_{h}^{2}(p u)+P_{E C-R}(p u) \sum_{h=1}^{h_{\max }} I_{h}^{2}(p u) h^{2}}{1+P_{E C-R}(p u)}=1
$$

Taking $I_{1}(p u)$ out of the summation in Eq. (12) results in:

$$
I_{1}^{2}(p u)+\left(\frac{\sum_{h=2}^{h_{\max }} I_{h}^{2}(p u)+P_{E C-R}(p u) \sum_{h=2}^{h} I_{h}(p u) h^{2}}{1+P_{E C-R}(p u)}\right)=1
$$

Or: 
$\sum_{h=2}^{h_{\max }} I_{h}^{2}(p u)\left(1+\frac{P_{E C-R}(p u) \times\left(h^{2}-1\right)}{1+P_{E C-R}(p u)}\right)=1-I_{1}^{2}(p u)$

The parameter $I_{1}$ is the rms value of the fundamental current. The right hand side of (16) determines the necessary decrease in load fundamental current in order not to let the winding hottest-spot temperature exceed its rated value. The decision of how much the load fundamental current must be lowered in order to keep the transformer life unchanged is taken by the customer. However, this can be used as a strategy to define a harmonic index in which harmonics have different weighting factors. If $P_{E C-R}(p u)$ is assumed to be $\alpha$, then:

$$
\frac{P_{E C-R}(p u)}{1+P_{E C-R}(p u)}=\frac{\alpha}{1+\alpha}=k
$$

From (16) and (17), the following harmonic index can be derived:

$$
W T H D=\sum_{h=2}^{h_{\max }}\left(1+k\left(h^{2}-1\right)\right) I_{h}^{2}(p u)
$$

In the new WTHD, the coefficient of each harmonic is equal to $1+\mathrm{k}(\mathrm{h} 2-1)$. Figure. 1 determines harmonic weighting factor as a function of:

$$
P_{E C-R}(p u) \text {. }
$$

For:

$$
P_{E C-R}(p u)=0.15 \text { : }
$$

Then:

$$
k=\frac{0.15}{1+0.15}=0.13
$$

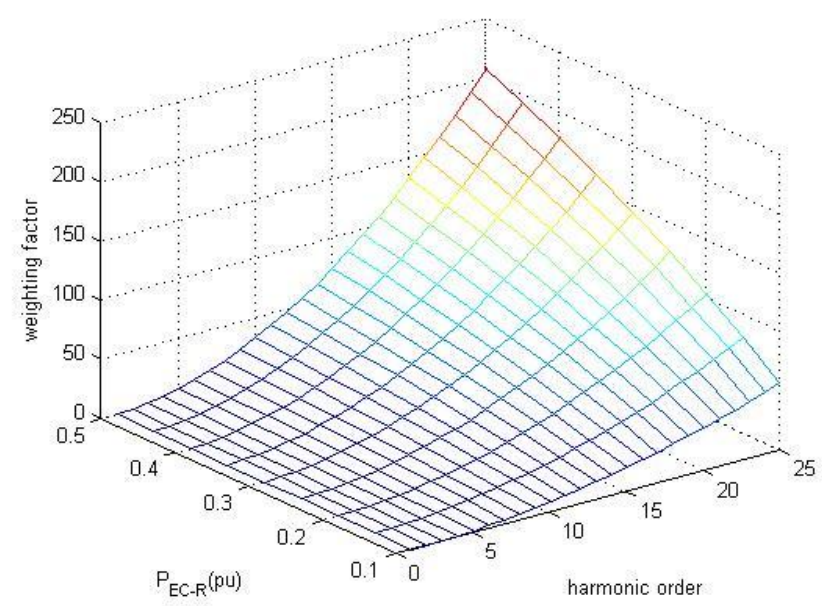

Fig.1. Harmonic weighting factors as a function of $P_{E C-R}(p u)$ and harmonic order.

The new WTHD index is then:

$W T H D=\sum_{h=2}^{h_{\max }}\left(1+0.13\left(h^{2}-1\right)\right) I_{h}^{2}(p u)$

Figure. 2 shows the coefficients for different harmonics if $P_{E C-R}(p u)=0.15$.

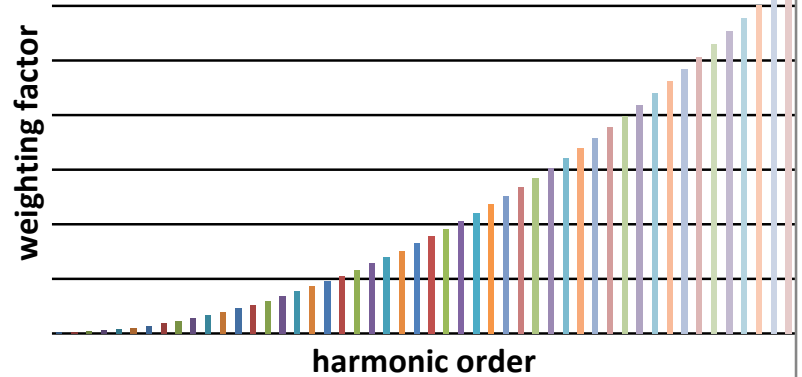

Fig.2. Harmonic weighting coefficients of individual harmonics

$$
\text { for } P_{E C}-R^{(p u)}=0.5
$$

\section{B. Liquid-Filled Transformers}

This section applies the same concept introduced in previous section to liquid-filled transformers. As expressed in [3], for a self-cooled ONAN mode transformer, the top oil rise over the ambient temperature is proportional to the total losses (including load losses and no-load losses) to the power of 0.8. Therefore;

$\theta_{T O}=\theta_{T O-R}\left(\frac{P_{N L}(p u)+\sum_{h=1}^{h_{\max }} I_{h}^{2}(p u)+P_{E C}(p u)+P_{O S L}(p u)}{P_{N L-R}(p u)+1+P_{E C-R}(p u)+P_{O S L-R}(p u)}\right)^{0.8}$

The hottest spot winding temperature rise over top oil rise temperature is proportional to the load losses to the power of 0.8 , i.e.:

$\Delta \theta_{H}=\Delta \theta_{H-R}\left(\frac{\sum_{h=1}^{h_{\max }} I_{h}^{2}(p u)+P_{E C-R}(p u) \sum_{h=1}^{h_{\max }} I_{h}^{2}(p u) h^{2}}{1+P_{E C-R}(p u)}\right)^{0.8}$

The life of a liquid-filled transformer is estimated using the hottest-spot winding temperature over the ambient temperature which is calculated by adding the winding hottest-spot temperature over top oil rise to the top oil rise temperature. In order to keep the transformer life unchanged when it is subjected to a harmonic load, the winding hottest-spot temperature over the ambient temperature must be held equal to its rated value. To simplify the problem, it is assumed that the winding hottest-spot temperature over top oil rise and the top oil rise do not exceed their rated values. In order to keep the winding hottest spot temperature over top oil rise equal or less than its rated value, load losses must not exceed their rated values. From Equation (12) :

$$
\left(\frac{\sum_{h=1}^{h_{\max }} I_{h}^{2}(p u)+P_{E C-R}(p u) \sum_{h=1}^{h_{\max }} I_{h}^{2}(p u) h^{2}}{1+P_{E C-R}(p u)}\right) \leq 1
$$

And as it was shown in previous section, the following condition must be held:

$$
\sum_{h=2}^{h_{\max }}\left(1+k\left(h^{2}-1\right)\right) I_{h}^{2}(p u) \leq 1-I_{1}^{2}(p u)
$$


Or:

$$
\sum_{h=1}^{h_{\max }}\left(1+k\left(h^{2}-1\right)\right) I_{h}^{2}(p u) \leq 1
$$

To keep the top oil rise unchanged, the same calculations as in the case of a dry-type transformer can be used. The only difference is that Eq. (22) must be used instead of (12). From (22), in order to keep the top oil rise unchanged, one has to show:

$$
\left(\frac{P_{N L}(p u)+\sum_{h=1}^{h_{\max }} I_{h}^{2}(p u)+P_{E C}(p u)+P_{O S L}(p u)}{P_{N L-R}(p u)+1+P_{E C-R}(p u)+P_{O S L-R}(p u)}\right)^{0.8} \leq 1
$$

Based on (7), if $\mathrm{h} 0.8$ is less than $(1+\mathrm{k}(\mathrm{h} 2-1)$, other stray losses will be less than its rated value if (24) is satisfied. In other words, if the harmonic conditions for the winding hottest-spot are held according to (24), there is no need to check the condition for the top oil temperature rise. This is true for $P_{E C-R}(p u) \geq 0.15$ and $\mathrm{h}>3$.

\section{Evaluating the New Harmonic Index Based on Real Measurements}

In order to show the effectiveness and suitability of the new proposed harmonic index, two different loads with almost the same THD are studied. Loads harmonic contents are measured by at the low voltage side of the distribution transformer by the use of a power quality analyser. The customers are located in a southern province of Iran. In both cases, the same distribution transformers with the parameters of $1600 \mathrm{kVA}, 20 \mathrm{kV} / 400 \mathrm{~V}, \mathrm{X}=6 \%$, $\mathrm{I}=1800 \mathrm{~A}$ is being employed. Tables I and II summarize the harmonic content of the loads, and Figures. 3 and 4 show the same results.

Table I: Harmonic content of Case No. 1

\begin{tabular}{|l|l|l|l|l|l|}
\hline $\mathrm{H}$ & $\mathrm{Ih} / \mathrm{I} 1$ & $\mathrm{H}$ & $\mathrm{Ih} / \mathrm{I} 1$ & $\mathrm{H}$ & $\mathrm{Ih} / \mathrm{I} 1$ \\
\hline 2 & $0.9 \%$ & 19 & $0.8 \%$ & 36 & $0.0 \%$ \\
\hline 3 & $4.3 \%$ & 20 & $0.0 \%$ & 37 & $0.4 \%$ \\
\hline 4 & $1.4 \%$ & 21 & $0.3 \%$ & 38 & $0.1 \%$ \\
\hline 5 & $10.7 \%$ & 22 & $0.1 \%$ & 39 & $0.0 \%$ \\
\hline 6 & $0.6 \%$ & 23 & $4.5 \%$ & 40 & $0.0 \%$ \\
\hline 7 & $6.0 \%$ & 24 & $0.0 \%$ & 41 & $0.3 \%$ \\
\hline 8 & $0.4 \%$ & 25 & $2.6 \%$ & 42 & $0.0 \%$ \\
\hline 9 & $0.8 \%$ & 26 & $0.2 \%$ & 43 & $0.1 \%$ \\
\hline 10 & $0.2 \%$ & 27 & $0.5 \%$ & 44 & $0.0 \%$ \\
\hline 11 & $3.5 \%$ & 28 & $0.2 \%$ & 45 & $0.0 \%$ \\
\hline 12 & $0.1 \%$ & 29 & $0.9 \%$ & 46 & $0.0 \%$ \\
\hline 13 & $1.5 \%$ & 30 & $0.0 \%$ & 47 & $0.3 \%$ \\
\hline 14 & $0.1 \%$ & 31 & $0.5 \%$ & 48 & $0.0 \%$ \\
\hline 15 & $0.2 \%$ & 32 & $0.1 \%$ & 49 & $0.2 \%$ \\
\hline 16 & $0.1 \%$ & 33 & $0.4 \%$ & 50 & $0.0 \%$ \\
\hline 17 & $1.6 \%$ & 34 & $0.1 \%$ & & \\
\hline 18 & $0.0 \%$ & 35 & $0.6 \%$ & & \\
\hline
\end{tabular}

Table II: Harmonic content of Case No. 2

\begin{tabular}{|l|l|l|l|l|l|}
\hline $\mathrm{H}$ & $\mathrm{Ih} / \mathrm{I} 1$ & $\mathrm{H}$ & $\mathrm{Ih} / \mathrm{I} 1$ & $\mathrm{H}$ & $\mathrm{Ih} / \mathrm{I} 1$ \\
\hline 2 & $0.2 \%$ & 19 & $0.0 \%$ & 36 & $0.0 \%$ \\
\hline 3 & $1.4 \%$ & 20 & $0.0 \%$ & 37 & $0.0 \%$ \\
\hline 4 & $0.1 \%$ & 21 & $0.0 \%$ & 38 & $0.0 \%$ \\
\hline 5 & $13.3 \%$ & 22 & $0.0 \%$ & 39 & $0.0 \%$ \\
\hline 6 & $0.2 \%$ & 23 & $0.0 \%$ & 40 & $0.0 \%$ \\
\hline 7 & $5.1 \%$ & 24 & $0.0 \%$ & 41 & $0.0 \%$ \\
\hline 8 & $0.1 \%$ & 25 & $0.0 \%$ & 42 & $0.0 \%$ \\
\hline 9 & $0.2 \%$ & 26 & $0.0 \%$ & 43 & $0.0 \%$ \\
\hline 10 & $0.0 \%$ & 27 & $0.0 \%$ & 44 & $0.0 \%$ \\
\hline 11 & $0.4 \%$ & 28 & $0.0 \%$ & 45 & $0.0 \%$ \\
\hline 12 & $0.0 \%$ & 29 & $0.0 \%$ & 46 & $0.0 \%$ \\
\hline 13 & $0.1 \%$ & 30 & $0.0 \%$ & 47 & $0.0 \%$ \\
\hline 14 & $0.0 \%$ & 31 & $0.0 \%$ & 48 & $0.0 \%$ \\
\hline 15 & $0.0 \%$ & 32 & $0.0 \%$ & 49 & $0.0 \%$ \\
\hline 16 & $0.0 \%$ & 33 & $0.0 \%$ & 50 & $0.0 \%$ \\
\hline 17 & $0.0 \%$ & 34 & $0.0 \%$ & & \\
\hline 18 & $0.0 \%$ & 35 & $0.0 \%$ & & \\
\hline
\end{tabular}

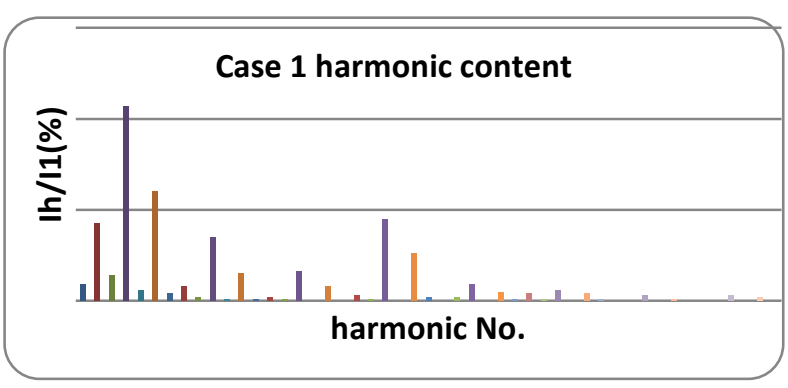

Fig. 3: Harmonic content of Case No. 1

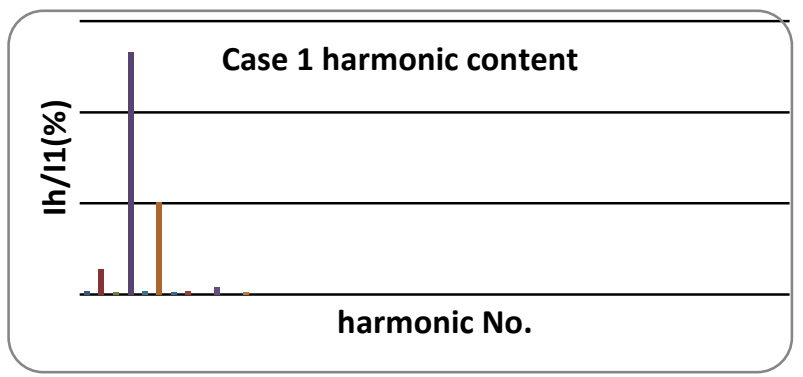

Fig. 4: Harmonic content of Case No. 2

From (8) and (9), the maximum current that the transformer in Case 1 can handle without overheating is $87 \%$. This value for Case 2 transformer is $97 \%$. In other words, Case 1 transformer has to be de-rated by $13 \%$ while Case 2 transformer has to de-rated by $3 \%$. $P_{E C-R}(p u)$ is assumed $15 \%$. Table III shows the THD and WTHD results for these two cases. As it can be seen, the two cases have almost the same THD level, while they are experiencing different harmonic effects. The Table shows that the new WTHD has been able to differentiate these two cases from harmonic effects point of view. 
Table II: Harmonic Indices for Cases 1 and 2

\begin{tabular}{|c|c|c|}
\hline & Case 1 & Case 2 \\
\hline THD & 14.84 & 14.33 \\
\hline WTHD & 15.8 & 1.6 \\
\hline
\end{tabular}

Another example is also given to show that the proposed WTHD is more effective than the conventional THD in terms of transformer effective life. The transformers have the parameters of $1200 \mathrm{kVA}, 20 \mathrm{kV} / 400 \mathrm{~V}, \mathrm{X}=5.7 \%$, $\mathrm{I}=900 \mathrm{~A}$. Harmonic contents of the two transformers are listed in Tables IV and V shown in Figures. 5 and 6.

Table IV: Harmonic content of Case No. 3

\begin{tabular}{|l|l|l|l|l|l|}
\hline $\mathrm{H}$ & $\mathrm{Ih} / \mathrm{I} 1$ & $\mathrm{H}$ & $\mathrm{Ih} / \mathrm{I} 1$ & $\mathrm{H}$ & $\mathrm{Ih} / \mathrm{I} 1$ \\
\hline 2 & $0.3 \%$ & 19 & $0.1 \%$ & 36 & $0.0 \%$ \\
\hline 3 & $0.6 \%$ & 20 & $0.0 \%$ & 37 & $0.0 \%$ \\
\hline 4 & $0.2 \%$ & 21 & $0.1 \%$ & 38 & $0.0 \%$ \\
\hline 5 & $7.6 \%$ & 22 & $0.0 \%$ & 39 & $0.0 \%$ \\
\hline 6 & $0.1 \%$ & 23 & $0.1 \%$ & 40 & $0.0 \%$ \\
\hline 7 & $2.2 \%$ & 24 & $0.0 \%$ & 41 & $0.0 \%$ \\
\hline 8 & $0.1 \%$ & 25 & $0.1 \%$ & 42 & $0.0 \%$ \\
\hline 9 & $0.3 \%$ & 26 & $0.0 \%$ & 43 & $0.0 \%$ \\
\hline 10 & $0.1 \%$ & 27 & $0.0 \%$ & 44 & $0.0 \%$ \\
\hline 11 & $1.2 \%$ & 28 & $0.0 \%$ & 45 & $0.0 \%$ \\
\hline 12 & $0.1 \%$ & 29 & $0.0 \%$ & 46 & $0.0 \%$ \\
\hline 13 & $0.7 \%$ & 30 & $0.0 \%$ & 47 & $0.0 \%$ \\
\hline 14 & $0.0 \%$ & 31 & $0.0 \%$ & 48 & $0.0 \%$ \\
\hline 15 & $0.1 \%$ & 32 & $0.0 \%$ & 49 & $0.0 \%$ \\
\hline 16 & $0.0 \%$ & 33 & $0.0 \%$ & 50 & $0.0 \%$ \\
\hline 17 & $0.1 \%$ & 34 & $0.0 \%$ & & \\
\hline 18 & $0.0 \%$ & 35 & $0.0 \%$ & & \\
\hline
\end{tabular}

Table V: Harmonic content of Case No. 4

\begin{tabular}{|l|l|l|l|l|l|}
\hline $\mathrm{H}$ & $\mathrm{Ih} / \mathrm{I} 1$ & $\mathrm{H}$ & $\mathrm{Ih} / \mathrm{I} 1$ & $\mathrm{H}$ & $\mathrm{Ih} / \mathrm{I} 1$ \\
\hline 2 & $0.1 \%$ & 19 & $0.2 \%$ & 36 & $0.0 \%$ \\
\hline 3 & $0.8 \%$ & 20 & $0.0 \%$ & 37 & $0.0 \%$ \\
\hline 4 & $0.0 \%$ & 21 & $0.0 \%$ & 38 & $0.0 \%$ \\
\hline 5 & $3.9 \%$ & 22 & $0.0 \%$ & 39 & $0.0 \%$ \\
\hline 6 & $0.0 \%$ & 23 & $0.5 \%$ & 40 & $0.0 \%$ \\
\hline 7 & $2.0 \%$ & 24 & $0.0 \%$ & 41 & $0.2 \%$ \\
\hline 8 & $0.0 \%$ & 25 & $0.1 \%$ & 42 & $0.0 \%$ \\
\hline 9 & $0.2 \%$ & 26 & $0.0 \%$ & 43 & $0.1 \%$ \\
\hline 10 & $0.0 \%$ & 27 & $0.0 \%$ & 44 & $0.0 \%$ \\
\hline 11 & $0.3 \%$ & 28 & $0.0 \%$ & 45 & $0.5 \%$ \\
\hline 12 & $0.0 \%$ & 29 & $0.0 \%$ & 46 & $0.2 \%$ \\
\hline 13 & $0.1 \%$ & 30 & $0.0 \%$ & 47 & $1.8 \%$ \\
\hline 14 & $0.0 \%$ & 31 & $0.0 \%$ & 48 & $0.1 \%$ \\
\hline 15 & $0.1 \%$ & 32 & $0.0 \%$ & 49 & $0.6 \%$ \\
\hline 16 & $0.0 \%$ & 33 & $0.0 \%$ & 50 & $0.2 \%$ \\
\hline 17 & $0.1 \%$ & 34 & $0.0 \%$ & & \\
\hline 18 & $0.0 \%$ & 35 & $0.0 \%$ & & \\
\hline
\end{tabular}

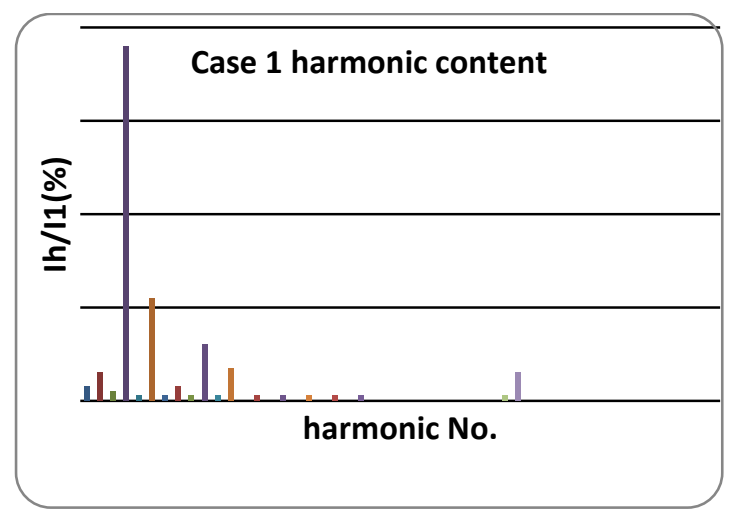

Fig. 5: Harmonic content of Case No. 3

Maximum currents that transformers in Cases 3 and 4 can handle without overheating are $98 \%$ and $94 \%$ respectively using (8) and (9). Therefore, transformers have to be derated by $2 \%$ and $6 \%$ respectively in order not to let the winding hottest spot temperature go over its rated value. $P_{E C-R}(p u)$ is assumed $15 \%$. Table VI shows the THD and WTHD results for these two cases. As it can be seen, although Case 4 transformer is experiencing a more severe case in terms of harmonic effects, its THD index is lower than that of the transformer in Case 3. Table VI shows that the new WTHD is effectively able to differentiate these two cases from harmonic effects point of view.

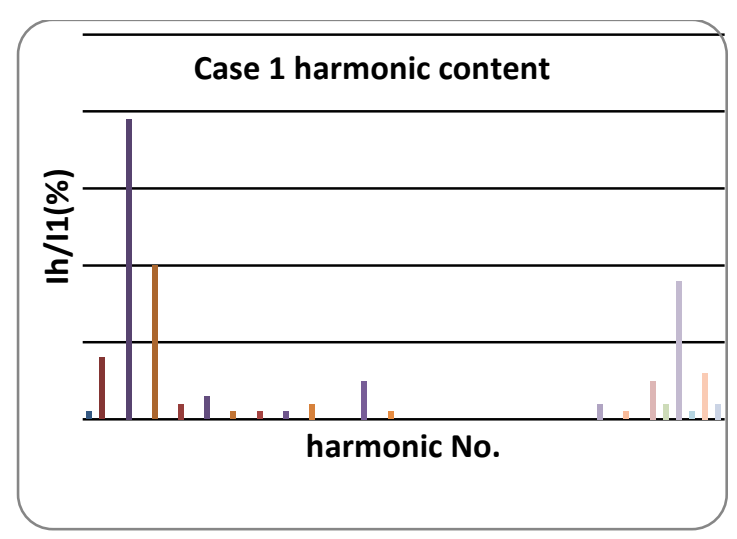

Fig. 6: Harmonic content of Case No. 4

Table IV: Harmonic Indices for Cases 3 and 4

\begin{tabular}{|c|c|c|}
\hline & Case 3 & Case 4 \\
\hline THD & 8.1 & 4.9 \\
\hline WTHD & 5.6 & 11.5 \\
\hline
\end{tabular}

\section{Transmission Losses}

This section studies the effects of higher transmission line losses caused by harmonics on the new proposed WTHD. Conductors used in distribution systems are usually composed of one circular conductor. Because of the skin effect, the ac resistance of the line is dependent on the frequency of the current, i.e. [6]:

$$
R_{a c}=R_{d c}\left(1+\frac{x_{s}^{4}}{192+x_{x}^{4}}\right)
$$


Where $R_{a c}$ is the line ac resistance, $R_{d c}$ is the line dc resistance, and

$$
x_{s}^{2}=\frac{8 \pi \cdot f \cdot k_{s} \cdot 10^{-7}}{R_{d c}}
$$

Where $\mathrm{f}$ if the system frequency in $\mathrm{Hz}, \mathrm{ks}$ is a factor determined by the conductor manufacturer, which is one for circular, standard, Compact and sectored lines. The following reasoning shows that if the WTHD for a drytype transformer is satisfied, resistive losses in a distribution line system will not exceed its rated value as well. For any value of $h$ between 2 , and hmax, it can be easily proved that the following non-equality is true:

$$
1+2 h^{2}<\left(h^{2}+2\right)\left(1+0.33\left(h^{2}-1\right)\right)
$$

To prove (30), this equation is simplified to:

$$
1+2 h^{2}\left\langle h^{2}+0.33 h^{4}-0.33 h^{2}+2+0.66 h^{2}-0.66\right.
$$

Rearranging (31) yields to:

$0.67 h^{2}<0.33 h^{4}+0.34$

which is true for $\mathrm{h}>1$.

Therefore, (32) is proved. Then a new factor $\mathrm{k}$ is defined as:

$$
k=\left(\frac{8 \pi f_{0} k_{s} 10^{-7}}{R_{d c}}\right)^{2}
$$

It is obvious that

$$
2 k^{2} h^{2}+192^{2}<\left(1+0.33\left(h^{2}-1\right)\right)\left(2 k^{2} h^{2}+192^{2}\right)
$$

If both sides of (34) are multiplied by $192 k$ and then are added to both sides of (23), again with a few changes one can write:

$$
\left(2 k h^{2}+192\right)(k+192)<\left(1+0.33\left(h^{2}-1\right)\right)(2 k+192)\left(k h^{2}+192\right)
$$

Or:

$$
\frac{\left(2 k h^{2}+192\right)(k+192)}{(2 k+192)\left(k h^{2}+192\right)}<\left(1+0.33\left(h^{2}-1\right)\right)
$$

Or:

$$
\frac{1+\frac{k h^{2}}{k h^{2}+192}}{1+\frac{k}{k+192}}<1+0.33\left(h^{2}-1\right)
$$

Therefore, if WTHD does not exceed its limit, then:

$$
\sum_{h=2}^{h_{\max }} I_{h}^{2} \frac{1+\frac{k h^{2}}{k h^{2}+192}}{1+\frac{k}{k+192}}<1-I_{1}^{2}
$$

Hence:

$$
\sum_{h=1}^{h_{\max }} R_{d c} I_{h}^{2}\left(1+\frac{k h^{2}}{k h^{2}+192}\right)<R_{d c} I_{\text {rated }}^{2}\left(1+\frac{k}{k+192}\right)
$$

The left side of (39) is the power loss in the line due to harmonics including the fundamental component, and the right hand side of (39) represents the rated power loss in the line. In other words, if WTHD does not exceed its limit in transformers, automatically power losses in lines will not exceed their rated value. Therefore, the new WTHD is only needed to be checked for the transformers.

\section{Conclusion}

In this paper, a new harmonic index is defined for current harmonics. The index is based on extra losses in a distribution system caused by harmonics. The idea is applied to dry-type and oil-immersed transformers and extended to transmission lines. The site measurements are studied, and it is shown that the current THD cannot effectively differentiate between the two cases. The new WTHD is able to discriminate the two cases. It is also shown that, from losses point of view, if the harmonic limits for transformers are not violated, there is no need to check the harmonic condition for transmission lines.

\section{References}

[1] IEEE (1992) Recommended Practices and Requirements for Harmonic Control in Electrical Power Systems, IEEE Standard 519-1992.

[2] IEEE (1995) IEEE Guide for Loading Mineral-OilImmersed Transformers, IEEE Standard C.57.911995.

[3] IEEE (IEEE1998) Recommended Practice for Establishing Transformer Capability When Supplying Non-sinusoidal Load Currents, IEEE Standard C57.110-1998 